\title{
A não realização do exame papanicolaou e comportamentos de risco em mulheres com vida sexual ativa
}

\author{
No papanicolous examination and risk behavior in women with active sex life \\ No realización del examen papanicolaou y comportamientos de riesgo en mujeres con \\ vida sexual activa
}

Ermínia Mayra Felix de Oliveira ${ }^{1}$, Silvia Ximenes Oliveira ${ }^{1 *}$, Mona Lisa Lopes dos Santos Caldas ${ }^{1}$, Moisés Barbosa Oliveira ${ }^{1}$, Marisa Ximenes Oliveira ${ }^{2}$, Thoyama Nadja Felix de Alencar Lima'.

\section{RESUMO}

Objetivo: Descrever os motivos da não realização do exame Papanicolaou e o comportamento de risco em mulheres sexualmente ativas. Métodos: Trata-se de um estudo descritivo-exploratório com abordagem quantitativa, realizado com 100 mulheres assistidas pelo NASF em um município do interior da Paraíba. A coleta dos dados ocorreu entre agosto e outubro de 2019, por meio da aplicação de um questionário e analisados de acordo com a literatura pertinente associada a uma análise estatística descritiva. Resultados: A maioria das mulheres são jovens, em união estável com renda familiar abaixo de um salário mínimo. Tiveram início da atividade sexual antes dos 18 anos, com parceiro fixo. O motivo da não realização do exame citológico foi a vergonha. $84 \%$ já ouviram falar em HPV e $83 \%$ sabem a forma de transmissão. A maioria tem de 1 a dois parceiros sexuais e realizam o exame anualmente. Conclusão: Apesar das estratégias nacionais para aumentar a cobertura do exame, ainda existem dificuldades relacionadas a realização, como a vergonha e o medo, influenciando na baixa cobertura a nível nacional.

Palavras-chave: Saúde da mulher, Fatores de risco, Neoplasias do colo do útero.

\begin{abstract}
Objective: Describe the reasons for not having the Pap smear and the risk behavior in sexually active women. Methods: This is a descriptive-exploratory study with a quantitative approach, carried out with 100 women assisted by NASF in a city in the interior of Paraíba. Data collection took place between August and October 2019, through the application of a questionnaire and analyzed according to the relevant literature associated with a descriptive statistical analysis. Results: Most women are young, in a stable relationship with a family income below the minimum wage. They started sexual activity before the age of 18 , with a steady partner. The reason for not performing the cytological examination was shame. $84 \%$ have heard of HPV and $83 \%$ know the form of transmission. Most have 1 to 2 sexual partners and perform the exam annually. Conclusion: Despite national strategies to increase the coverage of the exam, there are still difficulties related to the exam, such as shame and fear, influencing the low coverage at the national level.
\end{abstract}

Keywords: Women's health, Risk factors, Uterine cervical neoplasms.

${ }^{1}$ Centro Universitário de Patos (UNIFIP), Patos - PB.

2 Universidade Estadual da Paraíba (UEPB), Campina Grande - PB.

*E-mail: silviaximeneso@gmail.com

SUBMETIDO EM: 6/2020

ACEITO EM: 7/2020

PUBLICADO EM: 9/2020 


\section{RESUMEN}

Objetivos: Describa las razones para no hacerse la prueba de Papanicolaou y el comportamiento de riesgo en mujeres sexualmente activas. Métodos: Estudio descriptivo-exploratorio con enfoque cuantitativo, realizado con 100 mujeres atendidas por NASF en una ciudad del interior de Paraíba. La recolección de datos tuvo lugar entre agosto y octubre de 2019, mediante la aplicación de un cuestionario y analizados de acuerdo con la literatura relevante asociada con un análisis estadístico descriptivo. Resultados: la mayoría de las mujeres son jóvenes, en una relación estable con un ingreso familiar por debajo del salario mínimo. Comenzaron la actividad sexual antes de los 18 años, con una pareja estable. La razón para no realizar el examen citológico fue la vergüenza. El $84 \%$ ha oído hablar del VPH y el $83 \%$ conoce la forma de transmisión. La mayoría tiene de 1 a 2 parejas sexuales y realiza el examen anualmente. Conclusión: a pesar de las estrategias nacionales para aumentar la cobertura del examen, todavía existen dificultades relacionadas con el examen, como la vergüenza y el miedo, que influyen en la baja cobertura a nivel nacional.

Palabras clave: Salud de la mujer, Factores de riesgo, Neoplasias del cuello uterino.

\section{INTRODUÇÃO}

O câncer do colo do útero (CCU) é considerado um problema de saúde pública mundial, pelo fato de ser a segunda neoplasia mais comum entre as mulheres e pelos crescentes números de casos que surgem anualmente com diagnóstico tardio; levando a altos índices de morbimortalidade de mulheres em todo mundo (CASARIN MR, PICCOLIJCE, 2011).

Os países em desenvolvimento são os que apresentam as maiores taxas de incidência desta neoplasia, com $80 \%$ dos casos (BRASIL, 2017). Segundo as estimativas do Instituto Nacional do Câncer (INCA), para o biênio 2018-2019, foram estimados 16.370 casos novos de câncer do colo do útero no Brasil para cada ano, com um risco aproximado de 15,43 casos a cada 100 mil mulheres. Ocupando a terceira posição no país e o quarto tipo mais comum na população feminina no ranking mundial (INCA, 2017).

Entre as regiões brasileiras, observa-se grande diferença entre as taxas de incidência do câncer do colo do útero. A região Norte é a primeira mais incidente entre mulheres (25,62/100 mil). As regiões Nordeste (20,47/100 mil) e Centro-Oeste (18,32/100 mil), ocupam a segunda posição. Já as regiões Sul (14,07/100 mil) e Sudeste (9,97/100 mil), ocupam a quarta posição (INCA, 2017).

O Papilomavírus Humano (HPV) é o principal fator etiológico do CCU bem como o patógeno viral sexual mais comum (YIN F, et al., 2017). A descoberta do HPV como causa necessária para o desenvolvimento deste tipo de câncer tem levado estudiosos e governo ao desenvolvimento de vacinas profiláticas contra 0 vírus HPV e a ampliação de programas de rastreio e controle do câncer (SOTO D, et al., 2017).

A etiologia do CCU está associada diretamente aos hábitos de vida, aos fatores ambientais e as baixas condições socioeconômicas. Muitos são os fatores de riscos existentes que podem ocasionar o câncer do colo do útero, dentre eles, o tabagismo, multiparidade, multiplicidade de parceiros sexuais, uso de anticoncepcionais orais, iniciação sexual precoce e baixa ingestão de vitaminas, porém, o principal agente causador é o Papiloma Vírus Humano (HPV) (LEITE MF, et al., 2014).

Como formas de prevenção primária, estão ações comportamentais como vacinação contra o HPV, uso de preservativo, redução do número de parceiros sexuais e atraso no início da vida sexual. Já como prevenção secundária, o exame citopatológico (CP) é o mais utilizado, pois o câncer de colo do útero tem progressão lenta e se descoberta a lesão pré-invasiva precocemente, a chance de cura é quase total (SILVA VCG, RESENDE CL, 2009).

Apesar de o exame preventivo ser uma atividade oferecida com periodicidade, sua realização vem apresentando certa resistência por parte de muitas mulheres que não se submetem ao exame por diversos motivos, tais como, vergonha, ausência de sintomas e esquecimento, sentimentos de medo, o que revela a influência dos aspectos psicossociais de prevenção desse tipo de neoplasia (NASCIMENTO LC, et al., 2012). 
Diante disso, o conhecimento das mulheres sobre os fatores causadores e a forma de detecção precoce do CCU torna-se indispensável e pode contribuir para que as mesmas possam participar das ações e decisões que afetam sua saúde, principalmente estimulando a identificação de sintomas e a procura pelo serviço de prevenção adequado (LEITE MF, et al., 2014).

Nesse contexto, o presente estudo tem como alvo as mulheres com vida sexual ativa acompanhadas pela equipe do Núcleo de Apoio à Saúde da Família (NASF), que dá suporte as Unidades de Saúde da Família, nível de atenção responsável pelo rastreamento do câncer de colo uterino. Diante destes fatos, surge as seguintes questões norteadoras: Qual o comportamento de risco para o desenvolvimento do câncer de colo uterino das usuárias assistidas pelo NASF? Quais os motivos da não adesão ao exame citológico?

Além disso, sabe-se que um dos fatores contribuintes para o desenvolvimento do câncer uterino é o histórico familiar. Mulheres com parentes de primeiro grau (mãe ou irmã) que tiveram câncer de colo do útero tem um risco de 2 a 3 vezes maior de desenvolver a doença, quando comparado com aquelas que não tem casos na família (ONCOGUIA, 2017). Esse é o principal motivo para o desenvolvimento deste estudo, uma vez que a vivência com o histórico familiar de câncer de colo de útero na família (avó, tia, prima) tem causado sofrimento familiar e pessoal, por saber que este tipo de câncer pode ser evitado se utilizadas as estratégias de prevenção e rastreamento eficazes.

A proposta deste estudo visa oferecer uma reflexão sobre o comportamento preventivo em relação ao câncer de colo uterino e perceber quais crenças individuais impedem ou motivam a prática da adesão às recomendações do Ministério da Saúde. Espera-se que esses conhecimentos auxiliem no planejamento dos serviços de saúde no âmbito da gestão e da assistência, e sirva de suporte para futuras pesquisas relacionadas aos aspectos comportamentais que tornam a mulher mais vulnerável a este tipo de câncer. Nesse sentido, o presente estudo teve como objetivo descrever os motivos da não realização do exame Papanicolaou em mulheres sexualmente ativas.

\section{MÉTODOS}

Trata-se de um estudo descritivo, de campo, com abordagem quantitativa. A pesquisa descritiva tem como propósito observar, descrever, explorar, classificar e interpretar aspectos de fatos ou fenômenos. Buscam-se a frequência, característica, relação e associação entre variáveis (DYNIEWICZ AM, 2009).

A pesquisa foi realizada na área de abrangência da Estratégia de Saúde, com os grupos de Hiperdia, de gestantes e da academia da cidade assistidos pelo NASF, em um município do interior da Paraíba.

A população constituiu-se de todas as mulheres assistidas pelo NASF. A amostra foi aleatória simples, por conveniência, mediante os critérios de inclusão e exclusão. Os critérios de inclusão foram: mulheres na faixa etária acima de 18 anos, ter vida sexual ativa e que estivessem presente no momento da pesquisa. Foram excluídas as mulheres que apresentassem algum distúrbio mental que inviabilizasse sua participação neste estudo e que se recusassem a responder ao questionário não assinando o Termo de Consentimento Livre e Esclarecido (TCLE).

As participantes foram informadas quanto ao objetivo do estudo, bem como sobre o comprometimento e sigilo das informações prestadas no ato da pesquisa. Após receberem todas as informações sobre a finalidade da pesquisa, as mesmas para participarem do estudo, assinaram o TCLE, totalizando 100 participantes.

Foi utilizado um formulário, elaborado pelas próprias pesquisadoras, para a coleta dos dados sociodemográficos, clínicos e epidemiológicos. A coleta de dados ocorreu entre agosto e outubro de 2019, através da aplicação do questionário de forma individual, na própria instituição, onde houve a explicação acerca da pesquisa, assegurando os esclarecimentos necessários, para o adequado consentimento, e de possíveis dúvidas referentes à linguagem/nomenclatura utilizada no questionário.

A compilação dos dados foi realizada no banco de dados do Microsoft Exceß. Para análise estatística e descritiva, os dados foram importados para o programa Statistical Package for the Social Sciences (SPSS) versão 25. 
A pesquisa foi encaminhada ao CEP da UNIFIP e obedeceu aos preceitos da Resolução nำ510/2016 do Conselho Nacional de Saúde, que regulamenta a pesquisa envolvendo seres humanos (BRASIL, 2016), tendo aprovação através do CAAE 20069419.3.0000.5181.

\section{RESULTADOS}

Os dados sociodemográficos evidenciam maior proporção na faixa etária de 26 a 40 anos (40\%), casadas/união estável (67\%), com grau de escolaridade no ensino médio (43\%), e renda familiar mensal menor que um salário mínimo (49\%). A maioria não é fumante $(91 \%)$, e não têm hábito de beber semanalmente (79\%) (Tabela 1).

Tabela 1 - Perfil sociodemográfico e hábitos de vida das usuárias atendidas pelo NASF, $n=100$.

\begin{tabular}{|c|c|c|c|}
\hline Variáveis & & $\mathbf{N}$ & $\%$ \\
\hline \multicolumn{4}{|l|}{ Idade } \\
\hline & De 18 a 25 anos & 19 & 19 \\
\hline & De 26 a 40 anos & 40 & 40 \\
\hline & De 41 a 50 anos & 26 & 26 \\
\hline & Acima de 50 anos & 15 & 15 \\
\hline & Não informado & 03 & 3 \\
\hline \multicolumn{4}{|l|}{ Estado civil } \\
\hline & Solteira & 19 & 19 \\
\hline & Casada/União Estável & 67 & 67 \\
\hline & Divorciada & 06 & 6 \\
\hline & Viúva & 03 & 3 \\
\hline \multicolumn{4}{|c|}{ Escolaridade } \\
\hline & Fundamental Completo & 35 & 35 \\
\hline & Ens. Médio & 43 & 43 \\
\hline & Ens. Superior Completo & 16 & 16 \\
\hline & Não Informado & 06 & 6 \\
\hline \multicolumn{4}{|c|}{ Renda familiar mensal } \\
\hline & < 01 salário mínimo & 49 & 49 \\
\hline & 01 salário mínimo & 35 & 35 \\
\hline & 1 a 2 salários mínimo & 14 & 14 \\
\hline & Mais de 3 salários mínimo & 02 & 2 \\
\hline \multicolumn{4}{|l|}{ Fumante } \\
\hline & Sim & 09 & 9 \\
\hline & Não & 91 & 91 \\
\hline \multicolumn{4}{|c|}{ Hábito de beber semanalmente } \\
\hline & Sim & 21 & 21 \\
\hline & Não & 79 & 79 \\
\hline
\end{tabular}

Fonte: Oliveira EMF, et al., 2019. 
No tocante aos antecedentes ginecológicos e sexuais, a menarca ocorreu em uma idade entre 13 a 15 anos (62\%) e o início da atividade sexual foi antes dos 18 anos (51\%). Quando questionadas sobre ter parceiros fixos ou não, $63 \%$ afirmaram que atualmente possuem parceiro fixo. Com relação ao método contraceptivo utilizado, $38 \%$ realizam o coito interrompido e o número de parceiros durante a vida na maior parte dos casos foi de 1 parceiro (42\%) (Tabela 2).

Tabela 2 - Distribuição dos antecedentes ginecológicos e sexuais das mulheres atendidas pelo NASF, n=100.

\begin{tabular}{lcc}
\hline Antecedentes ginecológicos e sexuais & $\mathbf{N}$ & $\%$ \\
\hline Idade da menarca & & \\
\hline 10 a 12 anos & 35 & 35 \\
13 a 15 anos & 62 & 62 \\
15 a 17 anos & 03 & 3
\end{tabular}

\section{Coitarca}

\begin{tabular}{lll}
\hline$<18$ anos & 51 & 51 \\
$>18$ anos & 49 & 49 \\
\hline
\end{tabular}

Possui parceiro fixo

Sim

63

63

Não

37

37

\section{Método contraceptivo utilizado}

$\begin{array}{llc}\text { Camisinha masculino-feminina } & 27 & 27 \\ \text { Contraceptivo oral } & 21 & 21 \\ \text { Camisinha e pílula } & 05 & 5 \\ \text { Injeção } & 05 & 5 \\ \text { Coito interrompido } & 38 & 38 \\ \text { Ligadura tubária } & 22 & 22 \\ \text { Implante de DIU } & 06 & 12 \\ \text { Nenhum } & 35 & 35\end{array}$

Fonte: Oliveira EMF, et al., 2019.

Ao serem questionadas sobre a realização do exame, a maioria das mulheres já havia realizado. Apurouse nas respostas que a realização se dá anualmente ou a cada dois anos, todavia existe uma parcela que realiza a mais de dois anos. Sobre o conhecimento acerca das funções do exame, predominou a proporção de mulheres com a resposta de que o exame serve para rastrear câncer de colo de útero. Dentre as dificuldades mencionadas, a vergonha foi a mais citada e dentre as mulheres pesquisadas, $42 \%$ tiveram 0 resultado de seus exames alterados (Tabela $\mathbf{3}$ ). 
Tabela 3 - Informações relacionadas à saúde e ao exame citológico pelas mulheres atendidas pelo NASF, $\mathrm{n}=100$.

\begin{tabular}{lcc}
\hline Informações & N & $\%$ \\
\hline Você já fez algum preventivo na vida? & & \\
\hline Sim & 94 & 94 \\
Não & 06 & 6 \\
\hline Razão para procura do exame & 65 & 65 \\
\hline Exame de rotina & 09 & 9 \\
Examinar sobre problemas de saúde & 21 & 21 \\
Prevenir câncer do colo uterino & 01 & 1 \\
Por orientações de amigos & 03 & 3 \\
Porque o Agente Comunitário de Saúde orientou & 01 & 1 \\
Outros & &
\end{tabular}

Frequência da realização do exame preventivo?

\begin{tabular}{lll}
\hline Anualmente & 76 & 76 \\
A cada dois anos & 15 & 15 \\
A cada três anos & 03 & 03 \\
Nunca realizou & 16 & 16 \\
\hline Dificuldades para realização do exame Papanicolaou & & \\
\hline Medo & 10 & 10 \\
Vergonha & 49 & 49 \\
Insegurança & 02 & 2 \\
Preconceito & 01 & 1 \\
Acho que vai doer & 05 & 5 \\
Falta de tempo & 04 & 4 \\
Não tenho dificuldades & 30 & 30 \\
\hline Já teve algum exame alterado? & & 42 \\
\hline Sim & 42 & 48 \\
Não & 48 & 10 \\
Não sei & 10 & - \\
\hline Total & 100 & \\
\hline Fon & & \\
\hline
\end{tabular}

Fonte: Oliveira EMF, et al., 2019.

Sobre o entendimento em relação ao comportamento de risco, as mulheres informaram que tiveram relações sexuais com dois ou mais parceiros no último ano, afirmam ter conhecimento sobre o HPV, a forma de transmissão, relatando as consequências caso vejam a se contaminarem e a forma de evitar, embora saibam como preservarem-se não fazem uso do preservativo (Tabela 4). 
Tabela 4 - Informações relacionadas ao comportamento de risco e conhecimento sobre o HPV, segundo as mulheres atendidas pelo NASF, $n=100$..

\begin{tabular}{|c|c|c|}
\hline Antecedentes ginecológicos e sexuais & $\mathbf{n}$ & $\%$ \\
\hline \multicolumn{3}{|c|}{ Número de parceiros nos últimos 12 meses } \\
\hline Um & 42 & 42 \\
\hline Dois & 24 & 24 \\
\hline Três ou mais & 34 & 34 \\
\hline \multicolumn{3}{|l|}{ Costuma proteger o sexo com preservativo } \\
\hline Sim & 36 & 36 \\
\hline Não & 64 & 64 \\
\hline \multicolumn{3}{|l|}{ Já ouviu falar sobre HPV? } \\
\hline $\operatorname{Sim}$ & 84 & 84 \\
\hline Não & 16 & 16 \\
\hline \multicolumn{3}{|l|}{ Para você como se pega o HPV? } \\
\hline Vida sexual & 83 & 83 \\
\hline Saliva & 01 & 1 \\
\hline Beijo & 05 & 5 \\
\hline Não sei & 11 & 11 \\
\hline \multicolumn{3}{|c|}{ O HPV traz alguma consequência ao longo da vida? } \\
\hline Câncer do colo do útero & 75 & 75 \\
\hline Morte por câncer & 13 & 13 \\
\hline Não sei & 12 & 12 \\
\hline \multicolumn{3}{|l|}{ Tem como evitar o HPV? } \\
\hline Sim & 89 & 89 \\
\hline Não & 11 & 11 \\
\hline Total & 100 & - \\
\hline
\end{tabular}

Fonte: Oliveira EMF, et al., 2019.

\section{DISCUSSÃO}

Os resultados revelam que a maioria das participantes estavam na faixa etária de 26 a 40 anos, período de vida que constitui fator de risco para desenvolvimento de câncer de colo uterino, ao passo que a doença incide em apenas uma pequena porcentagem de mulheres com menos de 30 anos (BRASIL, 2010).

No tocante à escolaridade, uma pesquisa demonstrou que a maior parte das entrevistadas tem baixo índice de escolaridade. Os autores ressaltaram que tal fator, traz dificuldades relacionadas às medidas preventivas e de promoção à saúde da mulher, pois quanto menor o índice de escolaridade, maior a dificuldade na compreensão da manutenção da saúde, voltada às medidas preventivas e de controle (CARVALHO BA, et al., 2015). Estudo realizado com mulheres na Índia, China, México, Rússia e África do Sul, evidenciou que mulheres com maior grau de escolaridade apresentam 10 vezes mais probabilidade em participar de programas de rastreamento do CCU em detrimento das demais (AKINYEMIJU T, et al., 2016). 
Uma pesquisa buscou avaliar o perfil sociodemográfico de mulheres usuárias do serviço de saúde. Os resultados apontaram que 57\% recebem de um a três salários mínimos e $30 \%$ até um salário mínimo. Estes resultados concordam com esta pesquisa, na qual se observou que todas as entrevistadas pertencem à classe econômica desfavorável. As mulheres com condições econômicas desfavoráveis tendem a procurar mais pelos serviços de saúde apenas para procedimentos curativos, não visualizando a importância da prevenção. Isto dificulta o processo de cobertura do exame, colaborando para uma baixa adesão e maior propensão no desenvolvimento de doenças (SILVA ECA, et al., 2015). Concernente ao estado civil, uma pesquisa demonstrou resultados semelhantes, apontando que $70 \%$ são casadas (SILVA JP, et al., 2018). Mulheres casadas tendem a procurar mais os serviços de saúde quando comparadas as solteiras e viúvas, conjeturase por possuírem vida sexual mais ativa, assim, a estabilidade conjugal pode ser um aspecto positivo na prevenção do CCU.

Apenas 09 mulheres da amostra eram tabagistas, o que contribui de forma positiva para a prevenção do $\mathrm{CCU}$, visto que o tabagismo influencia o aumento da incidência de Neoplasia Intra-Epitelial Cervical (NIC) e câncer cervical (CAUZ OR, et al., 2002). Com relação ao consumo de bebida alcoólica, 79\% negaram o uso. Segundo o INCA (2019), o consumo do álcool aumenta o risco de desenvolver diferentes tipos de câncer, assim, o fato das mulheres não consumirem bebida alcóolica reflete um fator protetivo contra o surgimento do CCU.

Os dados coletados permitem estimar que a maioria das participantes iniciou a atividade sexual antes dos 18 anos, ou seja, na adolescência, o que contribui significativamente para o aparecimento do CCU, devido à maior chance de contato com o HPV, que é o principal fator de risco para este câncer. De acordo com Duarte SJH et al. (2011), é nesta fase da vida que a cérvice não está completamente formada e os níveis hormonais estão desestabilizados, o que representa outro fator de risco para o surgimento do HPV, um dos principais causadores de câncer de colo do útero, quando não se utiliza preservative.

No presente estudo, $21 \%$ das participantes usavam anticoncepcionais orais. Quanto ao uso de anticoncepcionais orais como fator de risco para câncer de colo uterino, a literatura aponta que mulheres portadoras de HPV que usaram esse método contraceptivo por cinco a nove anos tiveram chance 2,8 vezes maior de desenvolver câncer do que as que nunca o utilizaram (ROSA MI, et al., 2009).

O presente estudo mostrou alta adesão a coleta de citologia oncótica entre as participantes e, sobre a periodicidade do exame, afirmaram fazê-lo anualmente. Concordando com os resultados deste estudo, um estudo relatou que $55 \%$ das entrevistadas não realizaram o exame por terem vergonha e $15 \%$ por terem medo. Os autores justificam que a vergonha se constitui um fator negativo na realização do exame, causando uma descontinuidade na assistência. Dada à exposição do corpo para o procedimento do Papanicolau, o sentimento de vulnerabilidade na exposição ao toque, e o julgamento do seu corpo por outra pessoa, remete ao sentimento constrangedor de invasão, tendo alguém desconhecido visualizando sua imagem corporal (SANTOS CM, et al., 2015).

No Brasil, a baixa adesão ainda é baixa, pois milhões de mulheres nunca realizaram o exame de rastreamento do CCU. SE o exame fosse realizado pelas mulheres no período preconizado, muitos óbitos poderiam ser evitados, uma vez que poderia ser realizado um tratamento precoce e adequado na identificação da doença, evitando a evolução desta malignidade causadora de mortes em todo o mundo (GONÇALVES TFP, et al., 2016).

Concernente a realização do exame citológico, vale salientar que neste estudo a maioria das mulheres realizou o Papanicolaou pelo menos uma vez. Esse fato contribui na detecção precoce do CCU, pois constituise de uma estratégia essencial para seu controle. No entanto, mesmo que haja uma baixa proporção de mulheres que não realizam o exame anual, este índice indica uma deficiência na prevenção, conforme preconizado pelo Ministério da Saúde. Para a Organização Mundial da Saúde (OMS) e o INCA, o exame deve ser realizado a cada três anos, após dois exames normais consecutivos realizados no intervalo de um ano. A repetição de um ano após o primeiro teste tem como objetivo reduzir a possibilidade de resultados falso negativos nessa primeira fase do rastreamento (INCA, 2016). 
O sentimento do medo provém de experiências negativas, tanto de terceiros como de sua vivência em coletas anteriores, além do medo da dor e do possível resultado positivo para o câncer. Esse sentimento, durante a coleta, faz com que algumas mulheres adiem a realização do exame preventivo, revelando a falta de informações sobre a importância do diagnóstico precoce, probabilidade de cura mais elevada e tratamentos mais sutis (SOARES MC, et al., 2010). Além disso, Pereira JS et al (2020) relata que apesar da amplitude do problema em questão, pouco tem se falado a respeito deste assunto entre as mulheres, e a falta de informação entre elas constitui uma das principais dificuldades que os profissionais de saúde encontram no combate ao CCU. Igualmente, das mulheres que realizam o exame citológico, poucas retornam na busca do resultado, o que dificulta na cobertura do rastreamento da doença.

A análise desses fatores se faz importante, pois podem atuar como obstáculos para um comportamento preventivo em relação ao CCU, impedindo o estabelecimento de ações eficazes para a prevenção. Assim, sua observação possibilita o direcionamento, por parte dos profissionais de saúde, de um caminho estratégico que permita o estabelecimento de intervenções focadas em reduzir as barreiras e iniquidades no acesso ao exame (AGUIAR RP, SOARES MC, 2015). Em relação ao número de parceiros, $42 \%$ das mulheres, afirmaram ter tido apenas 1 parceiro, sendo este o perfil predominante. Existe uma tendência de solteiras sem parceiros fixos constituírem um fator de risco de aumento na predisposição para o desenvolvimento do CCU, pela multiplicidade de parceiros sexuais (MOURA ADA, et al., 2010).

Sobre o comportamento de risco e o conhecimento sobre o HPV, nota-se que $84 \%$ afirmaram ter ouvido falar sobre o HPV. A maioria das mulheres afirmou positivamente quando questionada sobre conhecer o HPV, corroborando com os achados de Soares MBO, et al. (2020), em que a maioria das mulheres apresentaram conhecimento adequado. Os achados deste estudo foi diferente com o descrito na literatura, como, por exemplo o estudo de Francis AS et al. (2010), em que avaliaram o conhecimento de um grupo de mulheres na cidade de Johanesburgo, África do Sul e verificaram que a maioria não conhecia ou tinha ouvido falar sobre o HPV. No Brasil, estudo desenvolvido por Osis MJD et al. (2014) também relatou desconhecimento das mulheres com relação ao HPV e evidenciaram que menos da metade da população estudada tinha conhecimento adequado.

Vale salientar que mesmo que as mulheres deste estudo tenham relatado ter conhecimento sobre o HPV, parte delas já tiveram seus exames alterados. Essa desproporção pode ser evidenciada que, após o exame alterado, o profissional de saúde tenha explicitado para as mulheres sobre o HPV. No estudo de revisão integrativa realizado por Carneiro CPF, et al (2019) as atribuições do enfermeiro são relevantes em todo o processo do cuidar, desde a prevenção primária através de uma educação em saúde contínua, como na secundária através do rastreamento do diagnóstico de lesões precursoras pelo exame de citopatologia oncótica, pois os fatores de risco podem influenciar no processo de desenvolvimento do CCU. Saber o modo de transmissão do HPV poderia estabelecer novos comportamentos tanto preventivos quanto terapêuticos. Observou-se que $83 \%$ das mulheres tinham consciência da transmissão por via sexual; afirmaram que o HPV traz consequências ao longo da vida (75\%) e se tem como evitar (89\%). Também foram relatadas formas inadequadas de transmissão, que fogem ao espectro do HPV, tais como beijo e saliva.

\section{CONSIDERAÇÕES FINAIS}

O perfil do grupo de sujeitos estudado deve ser estimulado a participar das iniciativas de controle do câncer de colo do útero: mulheres com idade entre 25 e 64 anos e com vida sexual ativa, tendo sido pertinente a abordagem dessas participantes. Observamos que, apesar do conhecimento superficial, algumas mulheres realizavam o exame anualmente, uma parcela apresentou resultados alterados. Embora relatem conhecimento sobre o HPV, a maioria não costuma proteger o sexo com o preservativo. Dentre os motivos da não realização do exame citopatológico, a vergonha e o medo foram os mais citados. Nesta lógica, os profissionais de saúde devem fortalecer a prática da educação continuada, por meio de palestras coletivas e individuais e orientações sobre o comparecimento das usuárias à unidade de saúde. Assim, torna-se necessário o estabelecimento de estratégias que visem à realização do exame em períodos adequados, como forma de promover saúde e reduzir os índices de morbimortalidade por esse agravo, de acordo com a necessidade da paciente e também de estudos mais aprofundados quanto a esse aspecto. 


\section{REFERÊNCIAS}

1. AGUILAR RP, SOARES DA. Barreiras à realização do exame Papanicolau: perspectivas de usuárias e profissionais da Estratégia de Saúde da Família da cidade de Vitória da Conquista-BA. Rev Saúde Coletiva, 2015; 25(2): 359-379.

2. AKINYEMIJU T, et al. Life-course socioeconomic status and breast and cervical cancer screening: analysis of the WHO's Study on Global Ageing and Adult Health (SAGE). BMJ Open, 2016; 6:1-10.

3. BRASIL. Conselho Nacional de Saúde. Resolução n. 510 de 07 de abril de 2016. Trata de pesquisas e testes em seres humanos. Brasília: Diário Oficial da União, 2016.

4. CARNEIRO CPF, et al. O Papel do enfermeiro frente ao câncer de colo uterino. Revista Eletrônica Acervo Saúde, 2019; (35), e1362.

5. CARVALHO BA, et al. Exame Papanicolau: percepção de acadêmicas de enfermagem do Vale do Paraíba. Rev Eletr Enferm Vale do Paraíba,2015; 1(8): 43-62.

6. CASARIN MR, PICCOLI JCE. Educação em saúde para prevenção do câncer de colo do útero em mulheres do município de Santo Ângelo/RS. Ciênc. Saúde Coletiva, 2011; 16(9): 3925-3932.

7. CAUZ OR, et al. Co-fatores do HPV na oncogênese cervical. Rev Assoc Med Bras, 2002; 48(1): 73-78.

8. DUARTE SJH, et al. Fatores de Risco Para Câncer Cervical em Mulheres Assistidas Por Uma Equipe de Saúde da Família em Cuiabá, MT, Brasil. Ciencia Y Enfermeria XVII, 2011 (1): 71-80.

9. DYNIEWICZ AM. Metodologia da pesquisa em saúde para iniciantes. Difusão Editora, 2009.

10. FRANCIS AS, et al. Examining attitudes and knowledge about HPV and cervical cancer risk among female clinic attendees in Johannesburg, South Africa. Vaccine 2010; 28(50):8026-8032.

11. Gonçalves TFP, et al. Reflections on nurses' role and actions of public health to prevent cervical cancer. J Nurs UFPE on line, 2016; 10(6): 2214-2222.

12. INCA. Instituto Nacional de Câncer. Diretrizes Brasileiras para o Rastreamento do Câncer do Colo do Útero. Rio de Janeiro; 2016.

13. INCA. Instituto Nacional de Câncer. Estimativa 2018: incidência de câncer no Brasil/ Instituto Nacional de Câncer José Alencar Gomes da Silva. Coordenação de Prevenção e Vigilância. - Rio de Janeiro: INCA, 2017.

14. INCA. Instituto Nacional de Câncer. Causas e Prevenção: Bebidas Alcoólicas. Brasília (DF): Ministério da Saúde; 2019.

15. LEITE MF, et al. Conhecimentos e prática das mulheres sobre câncer de colo do útero de uma unidade básica de sáude. Journal of Human Growth and Development, 2014; 24(2): 208-213.

16. MOURA ADA, et al. Conhecimento e motivações das mulheres acerca do exame de Papanicolaou: subsídios para a prática de enfermagem. Rev Rene, 2010; 11(1): 94-104.

17. NASCIMENTO LC, et al. Conhecimento cotidiano de mulheres sobre a prevenção do câncer de colo do útero. Rev. Enferm. UERJ, 2012; 20(4): 476-480.

18. OSIS MJD, et al. Conhecimento e atitude de usuários do SUS sobre o HPV e as vacinas disponíveis no Brasil. Rev Saude Publica, 2014; 48(1):123- 133.

19. PEREIRA JS, et al. Tecnologia no cuidado como instrumento de diagnóstico do câncer no colo do útero: um relato de experiência. Revista Eletrônica Acervo Saúde, 2020; (42), e2260.

20. ROSA MI, et al. Papilomavirus e neoplasia cervical. Cad Saude Publica, 2009; 25(5): 953-964.

21. SANTOS CM, et al. O enfermeiro na assistência à mulher com câncer de colo uterino. Rev Científ Enferm, 2015; 5(14): $19-24$.

22. SILVA VCG, RESENDE CL. Adesão das acadêmicas de enfermagem do centro universitário da grande dourados ao exame preventivo papanicolaou. Interbio, 2009; 3(2): 53-64.

23. SILVA ECA, et al. Conhecimento das mulheres de 18 a 50 anos de idade sobre a importância do exame de Papanicolau na prevenção do câncer de colo uterino no município de Turvânia-GO. Rev Eletr Fac Montes Belos, 2015; 8(4): 99-202.

24. SILVA JP, et al. Exame Papanicolau: fatores que influenciam a não realização do exame em mulheres de 40 a 65 anos. Arq. Ciênc. Saúde, 2018; 25(2): 15-19.

25. SOARES MC, et al. Câncer de colo uterino: caracterização das mulheres em um Município do Sul do Brasil. Esc Anna Nery, 2010; 14(1): 90-96.

26. SOARES MBO, et al. Fatores associados ao conhecimento sobre papanicolaou. Cienc Cuid Saude, $2020 ; 19:$ e48557.

27. SOTO D, et al. Epigenetic Alterations in Human Papillomavirus-Associated Cancers. Viruses, 2017; 9(248): 2-18.

28. YIN F, et al. A novel trivalent HPV 16/18/58 vaccine with anti-HPV 16 and 18 neutralizing antibody responses comparable to those induced by the Gardasil quadrivalent vaccine in rhesus macaque model. Papillomavirus Research, 2017; 3: 85-90. 